August, but with no relief. The case now became of serions import, owing to the extreme exhaustion necessarily induced. On the last day of September my anxiety was somewhat relieved by the discovery of symptoms of threatened abortion. These symptoms continued; and, fortunately for the life of the mother, a foetus was thrown off on September 1st. Immedi ately afterwards, the more ungent symptoms, namely, the vomiting and dysentery, began to subside, but were replaced for a few days by retention of urine, for the relief of which the catheter had to be used at stated intervals.

By slow degrees the patient recovered. She has since been pregnant, and has given birth to a fullgrown child.

REMARKs. It is obvious that in this case the main point of practice lay in a correct diagnosis. Vomiting, in pregnancy, though a common symptom, and one which has been attended by even fatal results, as recorded in the experience of Drs. Montgomery, Marshall Hall, Haighton, Davis, Johnson, Burns, Churchill, Clay, and many other writers, is not often compli cated with dysentery. But that it may be thus complicated is certain from the above case: and this complication the older writers on midwifery have recognised and pointed out with much more of precision than our modern authors. Had I not diagnosed in this case the existence of the pregnant condition, I should have been bewildered by the symptoms, their intractable character under every reasonable variety of treatment, and their cause.

The second point to be considered is whether, under the circumstances, I ought to have endeavoured to produce abortion. This question must be discussed candidly.

It is quite clear that the natiral process of abortion did, in this case, save the woman's life; and this fact would, a priori, be in favour of such operation. I am, however, still undecided whether the operation is justifiable.

In 1852 a discussion took place in the Academy of Sciences, in Paris, regarding the propriety of inducing abortion in cases where the vomiting of pregnancy threatened the life of the mother; and opinions were then very fairly divided on the question. It seems, indeed, that in many cases where abortion is produced, death takes place as if from the shock of the operation, or from continuance of the symptoms; while in cases where abortion occurs naturally, the fatal result is more commonly prevented. It remains, therefore, an open question whether, in cases such as I have described, we ought to run the risk of operation, or whether we should await, even till death, the possibility of a relief of the symptoms by an action of the uterus itself to expel its contents.

The last point to be noticed relates to the medicinal and diatetic measures which should be employed. It is common sense itself that so long as the irritable cause remains, ordinary medicinal remedies can be of no avail. One common remedy, I mean opium, mav give temporary relief; but this is given at the risk of producing a preliminary excitement and subsequent exhaustion. When organic obstruction in the heart produces dropsy, what can the best of us do? I need not enforce the parallel. Indeed, I know of no remedy in the whole Pharmacopœia that can be considered effective so long as the uterine irritation remains. A variety of styptic remedies might of course be tried. They would in $\mathrm{my}$ opinion either have no effect, or increase the mischief.

Dr. Clay, whose name has but to be mentioned to ensure authoritative respect, suggests that in the vomiting of pregnancy, particularly in the form occurring during the latter months, the patient should be laid in the recumbent position, with the hips raised ; that concentrated food should be given in small quantities, at long intervals; and that if the os and cervix uteri were tender to the touch, a few leeches should be applied. This treatment has been attended with remarkable efficacy in some of his cases, and shoild another case such as I have de scribed happen in my practice, I should unquestionably carry out a treatment at once so rational, simple, and safe.

In my next excerpt I shall give the particulars of a case similar to the above, in which death took place fourteen days after abortion, from the continuance of intractable diarrhoa.

Royal Socisty. Of fifteen new Fellows of this Society elected on June 9th, the following six are members of the medical profession :-Henry J. Carter, Esq., Surgeon Indian Army, Bombay; William Bird Herapath, M.D., F.R.S.E., Bris tol; George Miurray Humphry, M.D., Cambridge; John Dennis Macdonald, Esq., Assistant.Surgeon Royal Navy; William Odling, M.B., Kennington Road, London; Thomas Watson, M.D., F.R.C.P., Henrietta Street, Cavendish Square.

\section{THE FORMATION OF CLOTS IN THE} VENOUS SYSTEM DURING LIFE.

(A THESIS FOR A MEDICAL ACT IN THE UNIVERSITY OF CAMBRIDGE.)

By GEORGE MURRAY HUMPHRY, M.D., F.R.S., Surgeon to Addenbrooke's Hospital; Lecturer on Surgery and Anatomy.

\section{I.-Formation of Clots in the Veins.} [Continued from page 583.]

THE extension of the clot in the direction of the heart is usually limited by the junction of some large vein which is sufficient to maintain the current in the main trunk. Often the clot does not reach quite so far as this. Thus, when the clot commences at the junction of the iliacs, on one side, it commonly extends about half way up the common iliac; and, in a case of cancer of the uterus, in which the iliacs, on both sides, were obstructed, the clot extended up the vena cava nearly as high as the renal veins. Sometimes the clot reaches further, and terminates in a round or conical end on the cardiac side of the point of junction of some large trunk. In the peripheral direction, it is prolonged to a variable extent into the tributary branches, but does not usually reach the small veins: indeed, the latter are very rarely obstructed, either primarily or secondarily, in this affection.

The clot not only fills and chokes up the vessel, so as to prevent the passage of blood through it, but also distends or stretches it; and this distension, together with a certain amount of irritation resulting from the presence of a solid body in its interior, soon produces an effect upon the walls of the vein, the results of which are exhibited chiefly, or almost exclusively, on the exterior of the vessel. Thus we soon find that there is inflammation of the investing cellular tissue, causing an effusion of serum, lymph, or pus; whereas, in the interior, there is commonly little change beyond a removal of the epithelium from the lining membrane, and more or less in. timate adhesion of the clot to it. There may be also an increase of redness at some parts, which is evidently due to staining by the contiguous blood, inasmuch as it is commonly proportionate to the colour of the contained clot, being deepest where the clot is darkest, and less marked, or quite absent, where the clut is composed chiefly of fibrine. 1 have never seen lymph or pus, or any inflarnmatory product, formed from the interior of a vein. This proves that the inner coats of veins are by no means easily excited to inflammation, and is quite in accordance with the results of experiments made upon the veins of animals by Lee,* Mackenzie,* and Virchow (Handbuch der Specielle Pathologie und Therapie, I, 161). It accords also with the general results of my experience, which by no means indicate a liability to inflammation in the inner coats of veins. I have, in many instances, applied a ligature to the chief vein of a limb after amputation, withou any ill result in a single case; and I have never seen any mischief caused by the ligature of a varicose vein or a hæmor rhoidal tumour, though I have employed that method of treatment very often. It is not improbable that where unfavourable symptoms have ensued in cases of this kind, they have been caused, not so much by inflammation of the vein itself, as by suppuration in the surrounding cellular tissue.

When examining a vein which is plugged by a tough and adhering coagulum, one can scarcely be persuaded that the circulation could ever have been re-established through it, if the patient had survived; yet there can be no doubt that this does take place, and that a vessel may, in process of time, resume its functions, and be restored nearly, if not entirely, to its natural condition, after its channel has been completely, or to a considerable extent, blocked up by a clot. The perfect restoration of the limbs in Case $I$, and in several other instances, assured me of this; and it is in accordance with the great difficulty which I have experienced in effecting the permanent obliteration of varicose veins by temporary ligatures, or by other means which frad for their object the formation of

* Medico-Chirurgical Transactions, $\mathrm{xxxv}$ and xxxvi. Dr. Mackenzie infers, from the results of numerous experiments on the venous system, that the origin of obstructive phlebitis is to be sought for in a vitiated state of the blood, that this causes an irritation of the lining membrane of the veins at various points, which, in tuin, leads to coagulation of the blood. I do not, however, discover sufficient evidence of this irritation of the lining mem brane of the veins, and think there are many reasons agaiust admitting that it is a necessary, or even the ordinary, intermediate link between the vitiated and the coagulated condition of the blood.

601 
coagula in the vessels. It appears that the blood is almost sure to revert to its natural channel, in process of time, unless the vein be completely destroyed.* The dissection in the following case illustrated the condition to which tire clots became reduced. A man, aged 63, died, of erysipelas and pleuropneumonia, ten days after resection of one ramus of the lower jaw, performed on account of extensive necrosis and suppuration, which had continued for several months, and had reduced him to a very low state. His health had long been bad; but he did not mention that he had suffered any particular affection of the lower limbs. I was led to examine the veins in consequence of the condition of the pulmonary arteries, presently to be described. In dissecting out the femoral and popliteal vein of the left side, I remarked that the investing layer of cellular tissue, usually so delicate, was more coarse, tough, and closely adherent, than natural. With this exception, there was nothing to attract attention on the exterior of the vessels, or in the structure of their walls. In the interior were numerous delicate, but tough, white bands or strings, extending across or along the vessels; some were adherent in their whole length, and others only at their ends; also small firm lumps of pale yel!'ow, or gravel, or golden colour, smooth on the surface, and more or less adherent to the inside of the vein. In some plac6.; there were merely yellowish stains in the lining membrane of the vein. The nature of these stains would have been scarcely recognisable, had they not been in most in. stances continuous with the threads or some other evident remains of the clots. These veins contained also coagula, which appeared to have been formed recently, probably after death. The popliteal and lower part of the femoral vein, on the right side, presented appearances similar to those on the left. The upper part of the femoral vein was occupied by a firm, dry, mottled clot; and the profunda was tightly plugged by a continuation of the same, of white colour. Above the junction of the profunda, the vein was distended by a clot of comparatively recent formation, which, in the centre, was semifluid and of dirty cream colour. 'This soft part contained red corpuscles, and larger pale cells having indistinct nuclei.

It is no uncommon thing for the middle part of the clot to be, as in this instance, softened and converted into a dirty pultaceous or creamy substance, in which corpuscles are found, varying in size and shape, less regularly formed than pus-cells, and having less distinct nuclei. These are intermixed with oil-globules and red corpuscles, which may be natural in appearance, or more or less misshapen and granulated, and in various stages of dissolution. The changes which the blood thus undergoes are, as it would appear from the experiments of Mr. Gulliver (Medico-Chirurgical Transactions, xxin, 138), similar to those which take place when it is subjected to con coction after its removal from the body. They seem most frequently to occur when the clot has been quickly formed.

In all the cases that I have seen, except one, the soft central part of the clot was walled in by the firmer exterior portion, so that there was no opportunity for any of the débris to enter the circulating current.

We come next to inquire what are the conditions of the blood which predispose it thus to coagulate in the veins during life. It has been already remarked, that the clots are most liable to form in persons who are in an enfeebled and cachectic state. Now, in this state, it is well known that the fibrine of the blood exceeds its normal proportions; and it appears that its tendency to coagulation is increased by there being also in the blood an cxcess of water, which dilutes the saline or ammoniacal elements, and thereby renders them less able to hold the fibrine in solution. Nevertheless, persons often remain in cachectic and anxmic states for a great length of time; and they may, in addition, suffer several and prolonged attacks of syncope, without any coagulation of the blood taking place. Indeed, the cases in which the latter occur's are quite the exception; and we therefore search for some other cause to explain the phenomenon in these exceptional instances. It is most frequent when the cachexia has been induced by some inflam matory affection; and we know that the effect of inflammation, more particularly when it attacks the serous membranes, is to increase the amount and the coagulability of the fibrine. The parturient state, which, especially in the early period of lactation, is productive of a similar effect upon the blood, is also marked by a great tendency to clotting of the blood in the venous system. The researches of Dr. Richardson (The Cause

* Hence in the treatment of varix, where it seems desirable to resort to perative procedure, my practice is to pass a needle or silver wire beneath the vein, and to allow the metal to find its way out by ulceration througl the ressel and the superjacent skin. of the Coagulation of the Blood: 1858) continued with great assiduity and care through a long period, give strong reason for his view that the fibrive is held in solution by the presence of ammonia; and that its tendency to coagulate in the body is increased, and its coagulation out of the body is accelerated, by a diminution of the volatile alkali of the blood; and it is quite probable that, in the cases which we are discussing, an insufficient quantity of this solvent medium is one of the proximate causes leading to the clotting of the blood in the vessels.* There may be, in addition, some alteration in those relations of the corpuscles to one another and to the walls of the vessels, which, though not well understood, have an important influence in facilitating the circulation of the blood.t Certainly there seems no good reason to attribute the affection to an introduction of pus or other morbid fluid into the circulating current.

The diagnosis of the disease is easy. Edema of the limb is often the first symptom. This may be attended with, or pre ceded by, uneasiness in the course of the affected vessels; and there is enlargement of the superficial veins, with, perhaps, induration of some of them. When the femoral vein is affected, the inner side of the thigh is sometimes swollen in a marked manner. The integuments usually remain white occasionally they are inflamed in patches, or in a more diffused manner; and occasionally they are mottled by purplish spots, like petechial spots, or like those resulting from ecchymosis. Mortification rarely or never results from this cause alone (Virchow, Handbuch der Speciellen Pathologie und Therapie, I, 171).

I have already said that the affection is rarely attended with any serious consequences. It does not commonly seem much to aggravate the patient's condition, or to diminish his chance of recovery: indeed, $I$ have sometimes observed an amelioration in the general condition of the patient to be coincident with the swelling of a limb which indicated an obstruction in the great vein; as though the general mass of the blood had become thereby relieved of a certain quantity of its redundant fibrine, and was consequently better fitted to minister to the healthy nutrition of the body. If the obstruction of a vein occur during the course of an inflammatory disease, it generally takes place when the disease is subsiding: it may, therefore, be regarded as an attendant on recovery, though it is an evidence of a low or cachectic state of system. I may again remark, that it seems to have no relation to the malady called "pyæmia"; and though necropsies prove that it is sometimes associated with the formation of clots in the pulmonary arteries I have not in any case had clinical evidence of its being followed by that formidable aflection.

Nevertheless, it is a dispiriting, tedious malady; it retards the restoration of the patient, leeps him confined to his bed, and causes much annoyance and apprehension. The liability to its occurrence is to be borne in mind as a reason against having recourse to depletion or purgation, or other measures which may exhaust the patient, or draw away the saline and watery ingredients of the blood, in the latter stages of an inflammatory or febrile affection. I have given ammonia in some cases in which I thought there might be a predisposition to the formation of clots; and, where the general condition of the patient is likely to be benefited by the use of such a medicine, we should not ignore the evidences which have been afforded of its influence in retarding coagulation of the fibrine. If given in a pure form, it is probable that some of it will enter the blood; and that it will operate in the living vessels, more or less, in the same manner as it is found to do when mixed with blood which has been removed from the body. When the blood has begun to clot in the veins of a limb, I do not think that much good results from any particular local treatment. The affection runs a certain course, and, if the disease upon

* The researches of Scherer and Lehmann (Physiological Chemistry, $i$ 97 ) show that the bluod sometimes exhibits an acid reaction in the puerperal state; the acid present is supposed to be the lactic. This may be associated with the fact that I have often observed the skin to be remarkably dry in cases in which the blood coagulated in the vessels during life. An incipient decompusition of the blood is regarded by Zimmermann to be one of the chief causes of its coagulation.

+ The commencement of the clots must, doubtless, be attributed to influences affecting the fibrine, rather than the corpuscles, because at the parts where they are first formed they are usually composed ulmost entirely of fibriue. Nevertheless, it is most probable that changes in one of the constituents of the blood are attended with corresponding alterations in the others; and the analogy drawn from what is observed in inflammation and in pregnancy, suggests that an increase in the coagulating tendency of the fbrine is accompanied by an increase in the adhesive qualities of the corpuscles, which would materially contribute to the formation of clots, and which may, indeed, sometimes be the immediately originating cause of them. 
which it is an attendant do not prove fatal, will gradually sub. side spontaneously; and the veins usually become clear again. Considering the nature and cause of the malady, we should be unwilling to resort to the use of leeches. Nevertheless, I have known decided relief follow their application when the pain and inflammation around the vein was considerable. Fomenta tions are sometimes attended with comfort.

Of the instances which $I$ have seen, the greater number have been in males.* Nevertheless, it appears to be the same condition of the veins which in women, after delivery, usually constitutes the disease known by the name of "phlegmasia dolens". The cause of the disease, and the results disclosed by dissection, appear to correspond very closely, whether it be developed after parturition, or in the course of an illness ; and the name "phlegmasia dolens in the male" has, accordingly, been applied to it by Sir H. Halford (Medical Gazette, x, 172) and others. l'hlegmasia dolens most frequently occurs after parturition at that time when the fibrine of the blood is found to be most abundant; viz., during the early period of lactation and it is most common in women who are weak at the time of delivery, or whose strength has been reduced by flooding, an especially in those in whom there has been, in addition, peri tonitis or some inflammatory affection of the chest. Mioreover, when a fatal result has followed, it has commonly resulted, not from the condition of the veins, but from some other cause.t It must, however, be observed that, in phleg masia dolens, the pain is usually more severe, and the disease, on the whole, is of a more acute nature than in the ordinary obstruction of the veins: it seems sometimes to originate in a morbid condition of the uterine veins, and is sometimes attended with, or productive of, those inflammatory and suppurative affections in distant parts which are attributed to a morbid condition of the blood.

Indeed, phlegmasia dolens would seem to occupy an intermediate position between the simple and comparatively innocent obstruction of the veins, which we have been considering, and the more severe and dangerous affection of the veins, which has been described by Arnott, ${ }^{+}$and others, as an occasional attendant upon wounds and injuries. 'The changes which occur in the veins, and in the blood contained in them, seem to be much the same in the three classes of cases, except that in the traumatic variety the inflammatory symptoms are com monly more severe. And there is the further important dif ference, that in it we are liable to encounter that alarming, and commonly fatal, train of symptoms which is supposed to depend upon a purulent infection of the blood; whereas, in the ordinary obstruction of the veins, as I have already said, such concomitants are little to be apprehended.

The purport of the foregoing remarks may be condensed into the following summary.

1. The great veins are very liable to become obstructed by clots forming in them when the patient is greatly debilitated, and when the circulation is enfeebled-by inflammatory affections, by discharging abscesses, difficult labours, and other causes.

2 . The clots result from an altered state of the blood, disposing the fibrine to solidify in those parts of the veins which offer the greatest facilities for its so doing.

3. The inflammation of the veins is a consequence of the presence of the clot, and is chiefly confined to their outer coats, and to the surrounding cellular tissue.

4. The clots may soften and become intimately connected with the walls of the vessels, and may lead to the complete and permanent obliteration of their canals: more commonly, however, they are removed, or shrink into delicate bands or fibres, which offer little or no obstruction to the circulation.

5. The affection rarely leads to any serious result. It may be associated with so-called pyæmia; but has no necessary or frequent connection with it.

\section{[To be continued.]} * Of forty non-puerperal cases tabulated by Dr. Mackenzie (Mcdico-Chirur-
gical Transactions, xxxvi, 235), about two-thirds were females, and one-third gical $T$ males.

+ See paper by Dr. 1)avis, who first pointed out the true nature of this disease, Medico-Chirurgical Transactions, vol. xii; also papers by other simps in his lactlons, colls atte in his lectures reetles phlegmasia dolens ite healthy.

\$ Medico-Chirurgical Transactions, xv, 46. Mr. Arnott remarks, and I think he was the first to call attention to the fact, that the iuflammatory changes " are usually limited by the passnge of a current of blood; where a (he entrance of a branch, and

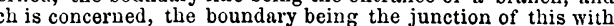
the trunk."
TWO ESSENTIALLY DISTINCT CONDITIONS OF KIDNEY GIVING RISE TO WHAT IS

\section{CALLED BRIGHT'S DISEASE.}

(ABSTRACT OF A THESIS FOR AN ACT KEPT IN THE UNIVERSITY OF CAMBRIDGE FOR THE DEGREE OF M.B.)

By W. H. Dicrissox, Caius College.

There are two essentially distinct conditions of kidney giving rise to what is called Bright's disease; and these may be distinguished from cach other by the presence or absence of cxternal granulation.

THE results of the author's minute examination of the kidney under disease, lead him to the conclusion that the conditions, called after the name of Dr. Bright, are capable of arrangement into two great classes; that in the one we have disease proper to the secreting surface of the tubes; while in the other the disease is essentially intertubular, and that the characteristic difference between the two is the presence in the latter, and not in the former, of external granulation.

The " tubular disease" includes all the varieties of the large smooth lidney; notwithstanding that pathologists have sub. divided it, according to the predominance of some one material, into "fatty degeneration," "waxy degeneration," "desquamative nephritis," and many others. There are two distinct stages. First, the period of enlargement; secondly, that of diminution. In the first stage, the capsule is thin and unadherent; the cortex is much, the cones slightly, enlarged; there is no appearance of preternatural vascularity. Parts, indeed, may be bloodless, giving a mottled aspect. The nicroscope shows the tubes entire; but in the cortex they are choked up with their epithelial secretion, which is often in various stages of disintegration, or fatty degencration. 'These are probably consequences rather than causes of the derangement, and result from the cells, formed in excess from the inflamed membrane, being of unhealthy composition. In the cones there is the same tendency to excess of epithelial secretion, but it finds a readier exit, and does not, therefore, so often accumulate there. The tubes here shew rather a tendency to become filled with a glassy fibrinous material, studded with specks of oil, or cells of epithelium, which happen to be passing down, or blood-corpuscles; and this is shed in the form of cylindrical casts. 'The casts, therefore, are formed almost exclusively in the straight tubes.

The second stage is rarely reached. It consists in an overstretching and bursting of the tubes one after another, those nearest the superficies being first destroyed; and it appears that their contents are then absorbed. The tubes collapse, and the organ may be reduced below its natural size. The capsule becomes thickened and a little more adherent. The surface is still smooth, the colour unchanged. A section shews the gland to be chiefly composed of cone substance, and the remaining cortical substance is coarse, pale, and firm in texture, being reduced more or less to its fibrous nature; but there is no evidence of an increase of fibrous matter. The author agrees with Mr. Simon in believing that the cysts, often present in this condition, are developed out of epithelial germs, which are left on the walls of the rupturea tubules.

The "intertubular," or " granular disease," commences with sligbt thickening and adhesion of the capsule, with processes of fibrous-looking material passing from it irito the in terior of the gland; contraction of these follows, the points at which they originate become lepressed, giving a superficial granulation to the exterior; and the tubes embedded in them, at first henlthy, become gradually reduced to microscopic threads.

In what the author calls "chronic intertubular disease" there is generally diminution of size. The adherent capsule has a tendency to split into two layers; the inner one consisting apparently of fibrioous effusion. 'The surface is granulated, and fibrous processes pass from the capsule between the granules. The fibrous tissue of the cortical part is increased, and the tubules waste. Some of the fibrinous material passes into the interior of the tubules, lining them and displacing the epithelium. Cysts are often present, and evidently result from transformation of the tubes, not, as in the tubular disease, from a change in the epithelium.

In the "acute intertubular disease" the granules are larger and more distinct, and there is more fibrinous exudation between and into the tubules.

The anatomical evidence in favour of the intertubular oricin of the discase may be summed up as follows :-

1. Near the capsule an effusion is often seen, obviously between the tubules. 